\title{
Interactive Evolutionary Computation algorithms applied to solve Rastrigin test functions
}

\author{
Yago Saez ${ }^{1}$, Pedro Isasi ${ }^{1}$ and Javier Segovia ${ }^{2}$
}

\{yago.saez|pedro.isasi\}@uc3m.es, fsegovia@fi.upm.es

${ }^{1}$ Department of Artificial Intelligence, University Carlos III of Madrid,

${ }^{2}$ Systems and Languages department, Faculty of Computer Science, University

Politécnica of Madrid,

Summary: this paper presents a new approach to interactive evolutionary computation that helps the user in the difficult task of finding an optimal solution between multiple possibilities. There are several ways of applying algorithms in interactive evolutionary computation; in this paper we explain three of them in order to make an experimental comparative study. Proceeding with a main goal of solving complex problems as fast as possible, we take the Rastrigin test function as a benchmark and it is executed with the three algorithms described. The aim is to show clearly the results of the algorithms in terms of solution quality and number of iterations. The results clearly show that the use of the proposed method based on chromosome learning heuristics works well even for non Interactive Evolutionary Computation frameworks ${ }^{l}$.

\section{Introduction}

The interactive evolutionary computation techniques have several applications such as the design (both artistic and functional) of 2D [1], and 3D objects [2]. Applications which make use of this technique for other practical purposes have also been developed such as the digital image processing [3,4,5,6], the composition of musical works [7], automotive design [8], the automatic design [9], general artistic design [10], the design of sculptures [11], and the generation of gestures in 3D figures aimed at virtual worlds [12].

\footnotetext{
${ }^{1}$ This research was supported in part by grants from MCyT project TRACER, Ref: TIC2002-04498$\mathrm{C} 05-04$
} 
In most of the above-mentioned references, the selection criteria are based on a merely artistic and personal point of view. In these kinds of applications the problems become more complex since the criteria used is subjective, (it is based on human perception and changes according to the user's opinions or personal preferences). This paper presents a non-subjective comparative method between three algorithms and an assertion of how increasing the selection pressure through the iterations makes it possible to overcome problems that arise when using micropopulations.

The remainder of the article is structured in the following way: a description of the algorithms used to compare the performance of our proposal, the description of the problem environment, the results of the tests, and finally, the conclusions drawn.

\section{Description of Algorithms}

IEC algorithms are difficult to test because they require the evaluation of the user in their experimentation. In addition, if we want to compare the presented algorithm with existing ones even more experimentation with humans is required. The proposed algorithm will be compared in every domain with the simple or classical GA (Simple Genetic Algorithm, SGA) and with a predictive fitness one, the Fitness Predictive Genetic Algorithm (FPGA). Through their variety and quantity the chosen algorithms offer a wide testing background. The SGA is always a good point of reference for the comparison and the predictive fitness algorithm is one of the latest and best proposals for the IEC algorithm [13].

The chosen representation for all the chromosomes is the same in all the contrasted algorithms. Each individual example is made up of various chromosomes which are in turn, made up of a vector of integers.

In the following section the function of the FPGA and the general details of its implementation for the experiments are described

\subsection{Fitness Predictive Genetic Algorithm}

Since the user is present during the evaluation and the selection process in IEC techniques it is necessary to work with micropopulations that are easily evaluated. A small number or a reduced population negatively affects the productivity of the GA's and as a result improvements to the conventional GA are proposed. These are based on the extension of the population and on the fitness prediction of all the individuals that are not evaluated by the user. [13,14]. Consequently, it deals with an algorithm of $M$ individuals out of which $N$ are shown to the user in order that he can make his evaluation. The fitness of the rest $(M-N)$ is obtained according to the differences with the selection made by the user in the previous iteration. There are 
several types of approaches to estimate the fitness of the rest, but we will only explain the most simple (Euclidean distance) in the following steps:

Step 1 - Initiate the population with $\boldsymbol{N}$ number of possible random solutions: during this process $N$ number of individuals is randomly initiated from the population. The value $N$ depends on the quantity of individuals that want to be shown to the user per iteration and it is also linked to the type of problem that wants to be solved. The experiments done have been based on $N=10$.

Step 2 - Evaluate $N$ candidates: this method is charged with the evaluation of $N$ visible candidates just as the user would do. As a result, and in the case that decoding was necessary, each of the genotypes are converted to corresponding phenotypes. With the fitness value or adaptation of each individual of the whole $N$ assigned, the determined evaluation function for each problem is applied. At this point, all other individuals of the population $(M-N)$ remain to be evaluated. In the experimental domain we work with populations of 100 individuals, out of which the user evaluates the best $10, M=100$ and $N=10$.

Step 3 - Forecast fitness values of candidates $M-N$ : this process is used in the cases in which the condition that guarantees the number of iterations is greater than 2. Alternatively, it could be said that it is used when an initial reference evaluation has been made which allows the forecasting of the fitness values of the rest of the population $(M-N)$. The necessary modifications to adapt the algorithm to the selection method have been done carefully and it corresponds to the proposals regarding the algorithm put forward by Hsu, [13].

Predictive fitness is obtained by the calculation of Euclidean distance between the referenced chromosomes (those selected by the user and those of the individuals not evaluated by the user)

FPGA's are effective in typical IEC problems in which the parameters are coded in such a way that the Euclidean distance gives an idea of how close or far an individual is form the one selected by the user. Given that the FPGA is not designed to be applied to numerical optimization problems and is not foreseen as being used with the proposed coding, it is possible that due to their genotype differences, two very close variables (as far as the phenotype is concerned) could produce great distances.

Step 4 - Select two parents: as with the SGA, the selection operator implemented for the experimentation is based on the selection of the two best individuals according to their adaptation or fitness value. However, the selection can only be done with the best ' $N$ ' individuals of the population. This previously mentioned limitation is typical of FPGAs and IEC techniques in which the user evaluates and, normally, the selection and in which he or she limits the evaluation to a sub whole of the population ' $N$ '. During each iteration, the selections of the user are stored in the $p s_{1}$ and $p s_{2}$ variables with the intention of doing other calculations that will obtain the predictive fitness of the following iterations. 
Step 5 - Cross pairs with parents: this process is exactly the same as the SGA. Once the best two individuals of fitness value are selected the cross operator is applied in order to obtain the new generation of individuals. The propagation strategy is elitist and, as a result, the selected parents go directly to the following generation and the rest are generated form the equally probable crossing of the parents.

Step 6 - Mutate the obtained descendants: as with the SGA, this process is responsible of mutating, with a certain probability, $\left(P_{\text {mutation }}\right)$ certain genes of the generated individuals. The objective is to guarantee the appearance of new characteristics in the following populations and to maintain enough genetic diversity.

Step 7 - Repeat until the final condition: like the SGA, the stop condition of the algorithm is imposed by a maximum limit of iterations.

\subsection{Proposed method: Chromosome Appearance Probability Matrix (CAPM)}

The proposed method is a genetic mutation one based on a matrix chromosome appearance probability (Chromosome Appearance Probability Matrix, CAPM), which is calculated according to the selections made by the user or the selection operator.

A series of modifications to the simple genetic algorithm (SGA) has been made for the development of the proposed algorithm that affects the operators of evaluation and selection and those of mutation. In addition, a new operator that eliminates identical individuals that appear has been included. All of these modifications result in increased computational costs which involve longer execution times. However, the algorithm is designed for the interaction of the user and consequently execution times are not a priority as long as they are reasonable. ( $<$ 2 seconds per iteration).

Step 1 - Initiate population and matrix probabilities MP: during this process all the individuals of the population are initiated randomly. In addition, the matrix of probabilities $M P$ is initiated through the equation (1).

$$
M P_{\text {gene }_{1}, \text { gene }_{2}, \ldots, \text { gene }_{l}}=\frac{1.0}{T}
$$

Where ' $l$ ' is the Number of genes that compose a chromosome of an individual and ' $T$ ' is the total Number of possible chromosomes calculated from the different genetic combinations in the equation (2).

$$
T=\prod_{i=1}^{l} \text { SizeGene }_{i}
$$


As a result, the previous equations take charge of realising an equally probable distribution between all the cells of the probability matrix producing a total sum of elements is equal to 1 . Each iteration involves the selection of two individuals and the combination of their chromosomes corresponds with one position in the matrix. Once the selection is made, the position that corresponds to each of the selected individuals is updated with a learning factor $\boldsymbol{\alpha}$.

Step 2 - Evaluate candidates: method responsible of evaluating the individual candidates just as the user would do.

Step 3 - Selection of parents: to alleviate, as far as possible, the effects of a small population, we would like to collect the preferences of the user and the selection operator in order to evolve generations orientated towards a solution that is based on their selections. Consequently, it has been decided to implement a structure capable of storing all the possible genetic combinations selected by the user. The simplest structure is based on a probability matrix $(M P)$ with as many dimensions as genes have chromosomes. During each iteration, user selections are stored in $p s_{1}$ and $p s_{2}$ variables and are used in subsequent processes in order to update the probability matrix, to execute the crossing of parents and to generate the descendant.

Step 4 - Update with $\alpha$ the probability matrix MP: after having made the selection, the probability matrix is updated with a value $\alpha$ which is called the learning factor. The probability matrix will update itself by multiplying the factor $\alpha$. In the same way to the probability updating rule of the PBIL algorithm it is necessary to normalize the probability matrix after having applied the updating rule. The determining of $\alpha$ comes from the experience acquired through several studies done with the incremental learning based in populations (PBIL) algorithm $[15,16]$, and the empirical prove that it functions. Nowadays we are developing the mathematical explanation about the optimum values of this factor. A small learning rate means that the algorithm makes a wider explorative search while a large learning rate means that the algorithm focuses on the search for a solution much quicker. However, a high learning rate means that the solution largely depends on the individuals selected during previous iterations.

Step 5 - Cross pairs with parents: this process is similar to that described for the FPGA. However, it incorporates a modification that affects the propagation strategy is no longer elitist. The cross operator is applied in order to obtain the new generation of individuals when the two individuals with the best fitness values have been selected.

This is a pseudo-elitist strategy which means that the selected parents pass to the following generation without any type of crossing. However, they are susceptible to modifications due to the mutation operator that is applied to them in the following step. The rest of the population is obtained from the equally probable crossing of both parents after the operator mutation has been applied. This pseudo- 
elitist strategy of propagation means that the user does not have to re-evaluate the individuals that he or she selected previously.

Step 6 - Orientated mutation of the obtained descendants: this is the process that marks the great difference between the proposed algorithm and the rest. It is responsible of the task of mutating, with a certain probability $\left(P_{\text {mutation }}\right)$, certain genes of the generated individuals. The aim is to guarantee the appearance of new characteristics in subsequent populations and to maintain a sufficiently wide genetic diversity that prevents premature convergence (something very common when working with small populations). However, the mutation in this algorithm does not mean a completely random modification of the value of the genes; it means a directional modification towards the tendency that the user or the selection operator has shown up until that moment. This knowledge is stored after the selections made in each iteration by the probability matrix $M P$.

For the mutation of each gene selected with a probability $P_{\text {mutation }}$ one chromosome is taken as a reference and serves as a pattern for the mutation process. This chromosome is obtained from all the ones possible by following the relative probability of the matrix $M P$. The higher the possibility of the appearance of a particular chromosome, the greater the possibility will be that it will be selected as the reference chromosome.

Step 7 - Look for and eliminate clones in the obtained descendants: in order to cover the maximum number of solutions it is necessary to take advantage of the diversity offered by the whole population when working with users and micropopulations $(<=10)$. In order to do this, and in accordance with the fact that small populations tend to converge prematurely (due to a lack of genetic diversity), an operation is carried out. This operator is capable of looking for and muting all those individuals that have an identical genetic code until a population without clones is guaranteed. This step avoids premature convergence by increasing genetic diversity, and avoids the appearance of various identical individuals in the same generation (clones).

In addition, it has to be kept in mind that the appearance of identical individuals begins to occur in direct relation is the gradual reduction of search space and the fact that, by that time, the matrix probability has already collected sufficient information regarding user preferences. As a result, the orientated mutation that occurs during the process of eliminating clones may positively affect the convergence of the algorithm. The 'elimination of clone operators' may also be useful when applied to other algorithms, but its fundamental problem is the execution time required for the search, compare and change numerous individuals.

Step 8 - Repeat until the final condition: as the rest of the algorithms to be compared, the stop or final condition of the algorithm is imposed by the maximum number of iterations. 


\section{Testing the algorithms: the Rastrigin function}

This testing function was initially proposed by Rastrigin in 1974 [17] limited to only 2 variables. This use was extended years later by Mulhlenbein, Schomisch and Born, [18] in order to allow the increase of the Number of variables to be optimized, just as is shown in equation (3).

$$
F 6(X)=10 * n \sum_{i=1}^{n} x(i)^{2}-10 \cos (2 \pi x(i))
$$

The equation represents a compilation of peaks and troughs with numerous local minima. This characteristic makes the function very complicated to optimize although it is an ideal test for genetic algorithms. The generalized Rastrigin function is a non-linear one, multidimensional, multimodal and quadratic with a minimum of zero at its origin. In the tests done, the search space is limited to -4.87 $<=x_{i}<=5.12$, with $i=1 . .20$ and $\Delta x_{i}=0.01$ on each axis. It has been decided to modify the search space of the original algorithm in order to adapt it to the encoding proposal for the algorithms. This means that the search becomes more complicated since it is done in an asymmetrical space of variables.

The proposed coding is based on a vector of three integers that can represent a total of 1000 different possible solutions, the total search space $n=10$ is formed by $1000^{10}=10^{30}$ possible solutions. With the algorithms proposed in the epigraph 2 and with the parameters shown in the following table, the Rastrigin function has been proved to minimize its result through 10 variables:

\begin{tabular}{lcccc}
\hline & SGA_100 & SGA_10 & FPGA_100 & CAPM_10 \\
\hline Population size & 100 & 10 & 100 & 10 \\
Iterations & 250 & 250 & 250 & 250 \\
Selection & 2 & 2 & 2 & 2 \\
Crossover & $50 \%$ & $50 \%$ & $50 \%$ & $50 \%$ \\
Mutation $\left(\mathrm{P}_{\mathrm{m}}\right)$ & $5 \%$ & $5 \%$ & $5 \%$ & $20 \%$ \\
Learning rate $(\alpha)$ & N/A & N/A & N/A & 0.001 \\
\hline
\end{tabular}

Table 1. Parameters used for the experiments

The mutation probability and the learning rate have been obtained after having done a detailed empirical study on each of the experiments for each one of the algorithms. 


\section{Results}

The results of the contrasted algorithms that aim to optimize the variables (which minimize the Rastrigin function) are collected in table 2. As can be seen in this table of the 4 algorithms compared, during the first 100 iterations the SGA 100 algorithm is the one that converges most rapidly and it is followed by the proposed CAPM 10 algorithm. This difference is due mainly to the fact that the size of the population negatively affects to the FPGA 100 and CAPM 10 algorithms, which have been designed for typical IEC problems, and in which only 10 individuals are evaluated (even if the FPGA 100 later works with 100 after having applied an automatic fitness to the remaining 90 individuals). In fact, the table also shows that the reduced size of the population also negatively affects to the SGA 10 and, as a result, it occupies the last place in the listing.

\begin{tabular}{r|rr|rr|rr|rr}
\hline & CAPM 10 & & SGA 10 & FPGA 100 & & SGA 100 & \\
\hline Iteration & Fitness & Solutions & Fitness & Solutions & Fitness & Sol. & Fitness & Sol. \\
\hline 0 & 134,12 & 0 & 136,65 & 0 & 136,54 & 0 & 105,08 & 0 \\
25 & 16,30 & 0 & 19,59 & 0 & 21,77 & 0 & 1,24 & 0 \\
50 & 4,64 & 0 & 8,89 & 0 & 9,83 & 0 & 0,53 & 0 \\
100 & 0,43 & 4 & 3,95 & 0 & 3,32 & 0 & 0,36 & 2 \\
150 & 0,18 & 28 & 2,39 & 0 & 2,30 & 0 & 0,36 & 6 \\
200 & 0,00 & 45 & 1,61 & 0 & 1,63 & 0 & 0,18 & 14 \\
250 & 0,00 & 83 & 1,63 & 0 & 1,19 & 0 & 0,18 & 17 \\
\hline
\end{tabular}

Table 2. Results of 100 experiments and 250 iterations

It is worth noticing that the good efficiency of the proposed CAPM 10 algorithm in comparison to its competitors. In the first of the two cases it is superior to its competitors every time, while in the second it is better, on average, from the $108^{\text {th }}$ iteration onwards. In addition, the proposed method reaches the optimum solution in the $83 \%$ of the experiments carried out, in comparison to the $17 \%$ seen in the experiments done with SGA 100.

\section{Conclusions}

The proposed method is a genetic algorithm with some important modifications that make it better for IEC problems and perhaps for other fields. Through the mutation operator it possesses an ability to propagate and transmit the user preferences to the following generations of individuals. This ability is still based 
on natural genetic evolution since the most spectacular improvements or changes of primitive humans happened without sexual reproduction and thus as a result of mutation and not only crossover.

After analyzing the results the following conclusions have been reached:

During the $100 \%$ of the experiments carried out the proposed CAPM 10 method is better than the rest of the algorithms that work with small populations (10 individuals).

In addition, in the proposed problem the CAPM 10 is from the $108^{\text {th }}$ iteration onwards capable of surpassing the SGA 100 (an algorithm that works with 100 individuals and whose efficiency is sufficiently well known).

In 250 iterations the CAPM 10 algorithm converges in the only optimal solution in $83 \%$ of cases in comparison with a $17 \%$ success rate achieved by the SGA 100 algorithm.

The FPGA 100 algorithm is the only one compared that is typical of IEC and the algorithm tested improves its results in $100 \%$ of the cases tested.

The results suggest the application of the proposed method to more problems in order to enable the study of its behaviour. It seems like an ideal method for solving problems that require work with small populations, whether that is due to the need for very complicated evaluation functions or due to the interaction of the user. Finally, keeping in mind that the test environment belongs to a complex numerical optimization environment not typical of IEC, it should be highlighted that the results are very satisfactory.

\section{References}

[1] Dawkins R. (1986) “The Blind Watchmaker", Longman Scientific and Technical, Harlow.

[2] Bentley P. (1999) "From Coffee Tables to Hospitals: Generic Evolutionary Design", Evolutionary design by computers, Morgan-Kauffman, pp. 405423.

[3] Ngo J. T. and Marks J. (1993), "Spacetime Constraints Revisited". Computer Graphics, Annual Conference Series pp. 335-342.

[4] Sims K., (1991) Artificial Evolution for Computer Graphics, Comp. Graphics,Vol. 25, No. 4, pp. 319-328.

[5] Sims K., (July 1994) Evolving Virtual Creatures. In Computer Graphics. Annual Conference Series (SIGGRAPH '94 Proceedings), pp. 15-22.

[6] Sims K., (1994) Evolving 3D Morphology and Behaviour Schemes. In Fogel, L. J. Angeline, P.J. and Back, T. Proc. of the $5^{\text {th }}$ Annual Conference on Evolutionary Programming, Cambridge, MA: MIT Press, pp. 121-129. 
[7] Moore, J. H. (1994) GAMusic: Genetic algorithm to evolve musical melodies. Software available in 'http://www.cs.cmu.edu/afs/cs/project/airepository/ai/areas/genetic/ga/systems/gamusic/0.html'

[8] Graf J., Banzhaf W (1995). Interactive Evolutionary Algorithms in Design. Proceedings of Artificial Neural Nets and Genetic Algorithms, Ales, France; pp. 227-230.

[9] Vico F.J., Veredas F.J, Bravo J.M., Almaraz J.,(1999) Automatic design synthesis with artificial intelligence techniques. Artificial Intelligence in Engineering 13, pp. 251-256.

[10] Unemi T. (2000) SBART 2.4: an IEC Tool for Creating 2D images, movies and collage, Proc. of the Genetic and Evolutionary Computation Conference Program, Las Vegas, pp. 153-157.

[11] Rowland D. (2000) Evolutionary Co-operative Design Methodology: The genetic sculpture park. Proc. of the GECCO Workshop, Las Vegas, pp. 7579.

[12] Berlanga A., Isasi P. Segovia J. Interactive Evolutionary (2001) Computation with Small Population to Generate Gestures in Avatars, Proceedings of the Genetic and Evolutionary Computation Conference (GECCO-2001), pp. 823-828.

[13] Hsu F.-C., Chen J.-S. (1999), "A study on multi criteria decision making model: Interactive genetic algorithms approach", IEEE Int. Conf. on System, Man, and Cybernetics (SMC99), pp. 634-639.

[14] Nishio K., Murakami M., Mizutani E., Honda N. (1995) "Efficient fuzzy fitness assignment strategies in an interactive genetic algorithm for cartoon face search”, In Proc. Sixth International Fuzzy Systems Association World Congress (IFSA'95), pp. 173-176.

[15] Baluja S., Pomerleau D., Jochem T. (1994) "Towards Automated Artificial Evolution for Computer-generated Images", Connection Science, Vol. 6, No. 2,3, pp 325-354.

[16] Baluja S., (1998) “Using Knowledge To Create Probabilistic Models For Optimization". http://citeseer.ist.psu.edu/602315.html, pp. 1-26.

[17] Törn A., Zilinskas A. (1989), "Global optimization", 0-387-50871-6, Springer-Verlag, New York, Inc.

[18] Mühlenbein H., Schomisch D., Born J. (1991) “The Parallel Genetic Algorithm as Function Optimizer", Parallel Computing, Vol. 17, No. 6,7, pp. 619-632. 